\section{Las matemáticas en}

\section{la ingeniería}

a través

\section{de la historia}

Revista Ciencia e lngenieria Neogranadina ISSN OI24SI70, N' 13 (P.jgs. 53 - 60)
MARTHA C. MELO DE ALONSO

\section{Resumen}

Qué mejor manera de ver las matemáticas que a través de la historia. En este artículo se hace un recorrido por las diferentes culturas, resaltando algunos hechos importantes realizados en ellas. Se presenta el nacimiento de la ingeniería en Colombia, más exactanente el de la Universidad Nacional de Colombia. En la tecnología, las matemáticas también tienen su aporte principalmente en la computación que da un fuerte empuje a las comunicaciones, las cuales avanzan a diario, por lo que se hace un breve resumen histórico desde el calculador binario hasta la banda ancha que es la tecnología de punta en las comunicaciones.

Palabras clave: matemáticas, calculador binario, inteligencia artificial, sistemas expertos, microcomputadores, banda ancha.

\section{Abstract}

What better way to see mathematics that through history. In this article a joumey is done through di-

Doceme Universidad Mititar Nueve Granodo. Jefe del Departomemto de Matembicas. 
fferent cultures, standing out some important facts fulfilled in then. The birth of Engineering in Colombia appears, exactly the one of the National University of Colombia. In technology. mathematics have also contributed, mainly in computers that strongly foments communications, which advance on a day to day basis. Therefore, a good history brief is made from the calculating bivernier to the broad band which is the latest technology in communications.

Key words: mathematics, binary computer, artificial intelligence, expert systems, microcomputers, broadband.

\section{CONCEPTO DE INGENIERÍA}

El tírulo de ingeniero se utilizó por prinera vez en la Edad Media aproximadamente entre los años 1000 y 1200. Las palabras "ingenio" e "ingenioso" provienen del latín "in generare", que significan "crear". Por tanto, la persona que creaba o diseñaba mácuinas de guerra (arietes, catapultas. torres de salto, etcétera) vino a ser conocida como el ingeniator o "ingeniero".

la ingeniería puede definirse como la "aplicación de los principios científicos para beneficio del hombre, creando medios para la satisfacción de necesidades sociales o individuales". Esto lleva a

\footnotetext{
El papuro Rhind es el nejor urigen de injornución acerea de lo motemolko eopría; es llamado asi en honor de Henry Rhmd quien compró el texto en Luxor en 1858 y lo vendio at museo Brianico. cn donde se exhibio pur primera vez. Este popiro copiado por unt escribo llanrado Ahines, doto de 1650 a.C. . aunque de acuerdo con el escritor, habio srdo lomado de int tratado escrito entre 2000 y 1800 a C. Et texlo comthene ceres de achento problemas e mclaye solucrones

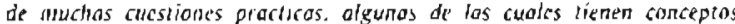

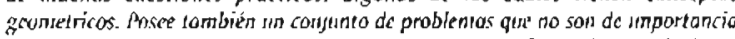
práctica y se freme la minoresión de que el autor los formuló y resolvib por diversion Lo publico por primero vez Eisenlohr en 1877

Exister otros cuatro escritos egercios mas cortos que son de importancia; al papiro de Moscí. al papuro de Kahun, el papuro de Berlin y el nollo de cueno El papiro de Moscu debe su nombre a lo ciudad en donde se encuentra. si comen2ó a descifrar du 1920 y cl documento compleio fue publicado en 1930 csle papiro contrene cerco de trinto problemas En 1900 un trambre trates de apellido Kohun descubrió ur popiro en el que se describion aphicaciones de los urélodos oritmétices descritos ell el popiro Rhind. Como con el paso del thempo cl rollo de eueno se secú y endureció completomente, se hrzo extremodomente difical desplegarto in destrum el texio. Los méindos quinticas modernos han permitido suavizado y prescrvarto y se exhibe en ul musco Britónico.
}

un compromiso óptimo entre economía, seguridad e información, para llegar al producto que satisface una necesidad humana.

Definición adoptada por el ICFES, ACOFI y S.C.l.:

"La Ingeniería es la profesión en la que los conocimientos de matemáticas y ciencias naturales, obtenidos a través del estudio, la experiencia y la práctica se aplican con juicio para desarrollar diversas formas de utilizar, de una manera económica, las fuerzas y materiales de la naturaleza en beneficio de la humanidad".

\section{CONTEXTO HISTÓRICO}

"Los pitagóricos consideraban la matemática como la cienciatipo del conocimiento; en este sentido, todo su sistemo tiendi

al matematismo que impregna la ciencia de hoy: $y$ asi ha podido decir Bertrand Russell que to más extraño de la ciencio moderna sca, quizá, sil vuclta al pitogorisıno".

Francisco Vera

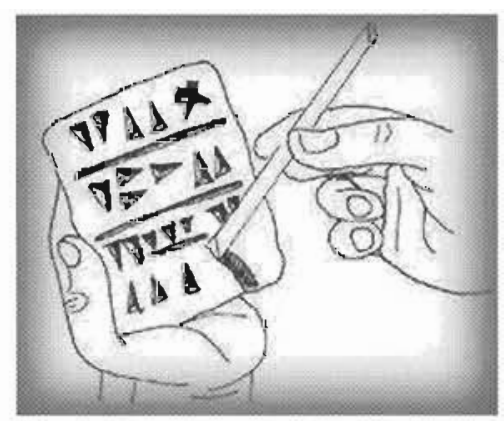

Figura 1

\section{MESOPOTAMIA Y BABILONIA}

En Mesopotamia, hacia el año 3200 a.C. , aparecen los primeros documentos escritos sobre tabletas de arcillas; se trata de textos contables en los que las cifras están representadas por muescas y las mercancías por picrogramas. Con los babilonios, las matemáticas alcanzaron un considerable desarrollo, dividieron la circunferencia en $360^{\circ}$, como producto de la observación del cielo y de los ciclos repetitivos. 


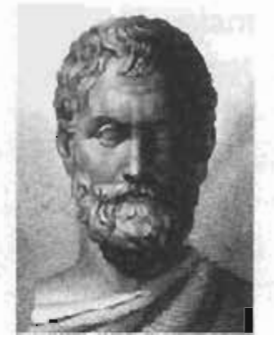

Figura 2. Toles de Mileio $(1630$ - 1550 a.C.).

\section{GRECIA}

Los primeros científicos de la historia que se educaron y trabajaron en las ciudades griegas, se preocuparon en especial por encontrar explicaciones racionales de los fenómenos de la naturaleza, pudiendo ser al misno tiempo matemáticos. geógrafos o biólogos, por lo cual los llamaban filósofos. El griego Tales de Mileto (1630-1550a.C.), como ingeniero dirigió obras hidráulicas y se dice que desvió el curso del río Halis mediante la construcción de diques; como astrónomo explicó los eclipses de sol y luna; el primer eclipse ocurrió el 28 de mayo de 585 a.C.. A los griegos en las matenúticas, se les debe el aporte de la abstracción geométrica, tal como la noción de "recta". Fueron los primeros en darse cuenta de que un enunciado matemático debía ser demostrado mediante la deducción lógica, a partir de ciertos hechos fundanentales llanados axionias y que no bastaba con ilustrarlos con un número bastante grande de casos en que se verificara. Son matemáticos famosos.
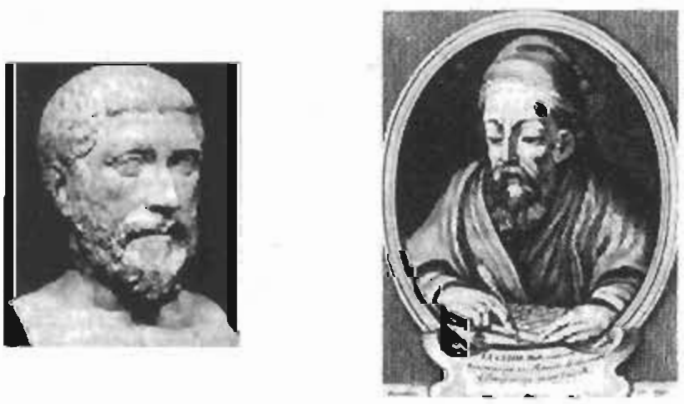

Figura 3. Pilágoras (582- 500 a.C) y' Findides (365- 300 a.C.).

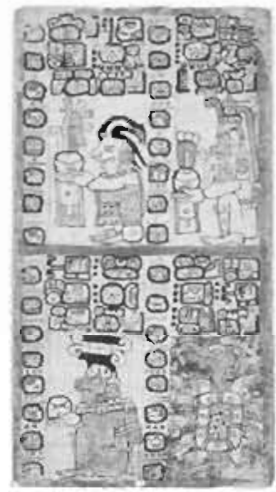

\section{EGIPTO $(3000-2500$ \\ a.C.)}

La matemática desarrollada en Egipto tenía un carácter práctico y servía como herramienta para el desarrollo y florecimiento de la civilización. Estaba soportada en dos principios fundamentales: el primero era el concepto del doble de un número y el segundo era la habilidad para encontrar los dos tercíos de cualquier número. Esto indica un manejo muy adecuado de las fracciones. elementos matemáticos fundamentales para el reparto, que era una de las formas importantes de manejo de recursos en la época en que el Río Nilo inundaba la tierra y se debía vivir del grano guardado. También eran útiles para el cobro de los impuestos de acuerdo con la clase social. Se tenía entonces la división de la unidad en fracciones unitarias (de numerador igual a 1), que sunladas daban cono resultado la totalidad.

Este carácter se observa tanibién en los desarrollos de geometria que sirvieron para hacer sus sorprendentes construcciones. Es bien sabido, a partir de la información encontrada en el famoso papiro Rhind (ver notal), que los egipcios calcularon el área del rectángulo tal cual se realiza hoy. De la misma forma calcularon el área del círculo con una fórmula sinuilar a la de hoy. Si se observa, estas figuras planas son los elementos fundamentales para construir un cilindro que era la forma de los silos en los cuales los egipcios guardaban el grano para la época en que no se podía 
cosechar. El problema 41 del papiro Rhind calcula el volumen de un granero cilíndrico.

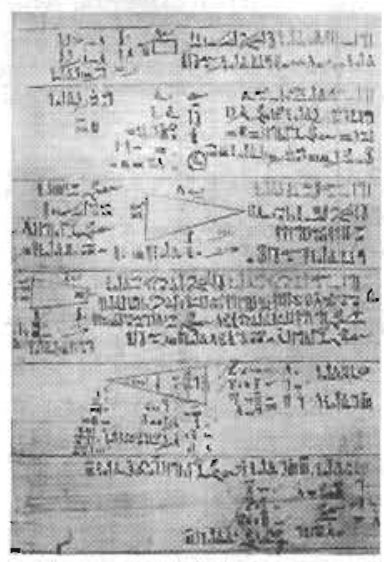

Figura 5. Popiro Rhind

\section{ISLÁMICA}

A principios del siglo VII, la ciencia y la técnica tuvieron un gran desarrollo en la civilización islámica. Los árabes inventaron los números dígitos y el sistema decimal, emplearon el cero y asignaron valor a los números, según el lugar que ocupaban.

A mediados del siglo XVIll, viene la segunda Revolución Industrial, época en que se registran avances en la ciencia y la tecnología, producidas por el desarrollo de las ciencias naturales, distinguiendo las siguientes áreas: noración matemática, teoría de las ecuaciones, descubrimiento de los logaritmos.

\section{LA INGENIERÍA}

La primera escuela de ingeniería que registra la historia es la École des Ponts et Chausées (Escuela de Puentes y Pavimentos) creada en Francia en 1794, aunque en 1646 ya se había instituido un cuerpo de ingenieros franceses de carácter militar.
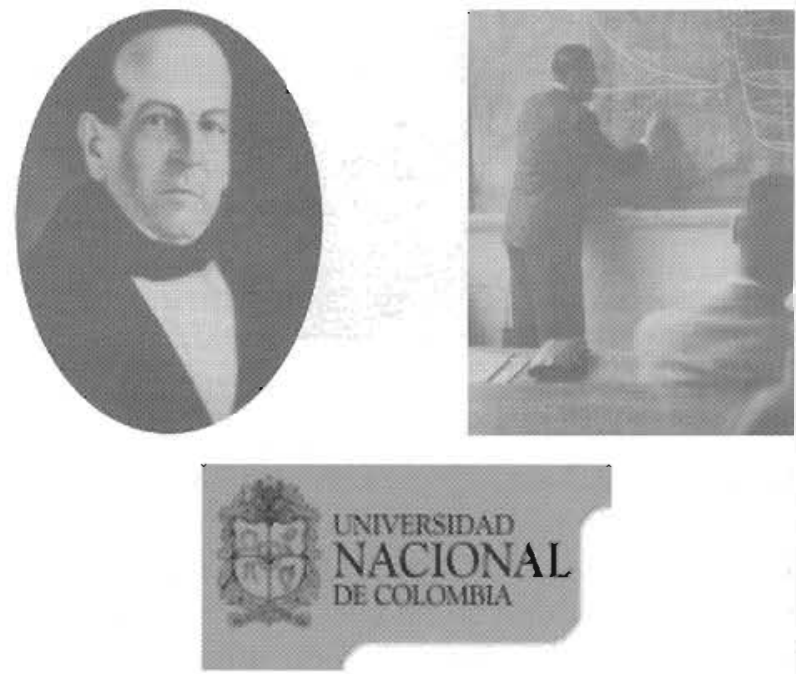

Figura 6. lino de Pombo (1797-1862) e ing. Arturo Ramirez Momúfor

\section{EN COLOMBIA}

En Colombia, pasado el período de la Independencia, la matemática se presenta con la creaciỏı del Colegio Militar (enero 2 de 1848), que tenía como misión prioritaria la formación de oficiales e ingenieros civiles. El profesor de los cursos de matemáticas lino de Pombo (1797-1862), estructuró la carrera de Ingeniería Civil con base en un núcleo básico de formación nratemática comenzando con los cursos más elementales de aritmética, geometría y álgebra, hasta los más avanzados que eran los de cálculo diferencial e integral. Así se graduaron los primeros ingenieros colombianos que fueron la primera semilla de la ingeniería civil en Colombia.

Con mucho pesar, el Colegio Militar es cerrado a finales de la década de 1860 , pero afortunadamente, durante el gobierno de Tomás Cipriano cle Mosquera, nació La Escuela Politécnica (1861), y más tarde la Universidad Nacional de los Estados Unidos de Colombia, el 3 de enero de 1868, cuando la Escuela Politécnica se anexa a ella con el nombre de Escuela de Ingeniería, heredando los profesores, los alumnos y los recursos que tenía, pero su consigna continuaba, siendo la de dar un buena formación matemática. 
A finales del siglo XIX, se plantéó la controversia sobre el tipo de formación matemática que debía darse a los ingenieros, pues había ursa cendencia y especial interés por lo práctico, lo técnico y lo productivo en lugar de lo formativo. Por lo que es creada la Escuela de Minas de Medellín en 1887, la cual se empeñó en darle un mayor énfasis a la matemática abstracta, hecho que también se dio en el programa de Matemáticas de la carrera de Ingeniería Civil de la Universidad Nacional, aunque no sobra comentar que este tipo de formación tuvo muchos enenigos. Discusión que se presenta en la actualidad ${ }^{6}$.

\section{LAS MATEMÁTICAS EN OTROS CAMPOS}

"Tampoco las matemáticas están por encima de las gentes concretas, de sus diferentes prejuicios, tabues y ensonaciones. A la postre las matemáticos hunden sus raices en los mismos magnas simbólicos en que se alimentan los mitos que aspiroba a desplazar. Cada matematica echa sus raices en los distintos imaginarios colectivos y se construye al hilo de los conflictos que se desatan entre los varios modos de representar / inventar esa ilusión que cada cultura llama realidad" Emmanuel Lizcano

Las matemáticas no son, pues la imagen estática ni el estereoripo de la perfección que no cambia. al contrario está aún llena de misterios cuyo esclarecimiento se demorará por siglos. Cada día las ciencias como la astronomía y la fisica le proponen problemas nuevos. También la ingenicría cuando trabaja con materiales transformados o elaborados por el hombre y elementos y procesos naturales de más dificil predicción y control (ríos, valles laderas costas etc.; lluvias, sismos inundaciones, etc.), deben ser modelados e interpretados por medio de planteamientos y análisis matemáticos, modelos fisicos, mediciones y ensayos de laboratorio.

También necesitan de las matenáticas todos los oficios. hasta los más humildes, el pintor de brocha gorda, la rejedora, el albañil, el cocinero, el sastre, etc. Cualquiera de estos oficios, sorpren- den por la multitud de datos numéricos, medidas y criterio geometrico que es posible aplicar en su desempeño. "Nada que parezca más alejado del rigor matemárico que el hacer un vestido. Los sastres ni se imaginan toda la geometría que podemos encontrar, si examinamos de cerca el problema, consistente en adaptar a la superficie curva del cuerpo humano, la superficie plana de una tela."7 De la misma forma, las matenáticas están en las ciencias humanas, como la economía, la sociología, el derecho la bistoria, la filosofía, etc., la nisma biología, entre las ciencias naturales, hoy lo utiliza como sucede ell biometría y genérica. Por ejemplo en la Universidad Militar Nueva Granada se hicieron y se llevan acabo investigaciones, usando la dimensión fractal, (valores dimensiónales entre el punto, la recta, el plano y el espacio. El fractal es un conjunto de puntos líneas o planos que se construyen a partir de iteraciones y que son semejantes bajo escala). Las investigaciones internacionales presentadas en la Universidad Militar Nueva Granada son: "Estimación de la dimensión fractal y su aplicación en fisica de suelos" y "Análisis fractal de la línea de base de macrófagos control y macrófagos infectados con Leishmania Amazonensis".

\section{LA TECNOLOGÍA}

El empleo de toda la amplia gana de métodos audiovisuales $y$ de programas de computador a nivel mundial ha penetrado la educación el todos sus niveles. El uso indiscriminado puede hacer que el objetivo del aprendizaje, cual es el de aprender el conocimiento básico y conceptual, se pierda "... los computadores solo manejan el nivel superficial de los sistemas matemáticos: los sistemas simbólicos. Los concepros que permiren utilizar los computadores y programas, programar los computadores, e interpretar los resultados impresos por ellos, no pueden estar en los computadores ni ser manejados por ellos: tienen que ser conscruidos por él mismo ... los compuadores no reemplazan la actividad mental de la construcción, exploración extensión y aplicación 
de los sistemas conceptuales. Si los computadores están disponibles, pueden apoyar maravillosamente esa construcción conceptual, pero si no están disponibles, hay muchas alternativas para producir los mismos efectos....".

\section{LA INFORMÁTICA}

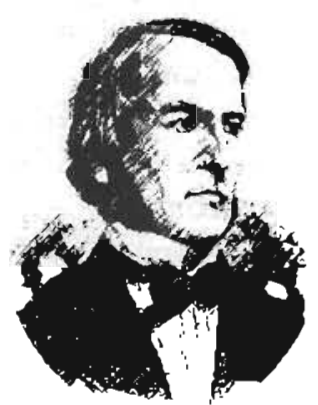

Figun 7. Ceorge Boole (1815 - 1864).

\section{CALCULADOR BINARIO}

El matemático norteamericano George R. Stibitz construyó el primer calculador binario, en los laboratorios Bell, en 1939, con el nonbre de model 1 relay comiputer o complex number calculator. Se trataba de un dispositivo lógico cuyos datos de salida representan la suma de los datos de entrada. En éste, el matemático usó relés telefónicos que funcionan segín el sistema del todo o nada (se usan sólo los números 0 y 1) con el fin de fabricar un calculador universal (en 1859 George Boole, lógico y matemático autodidacta inglés, había desarrollado la lógica simbólica, más específicamente las operaciones de la lógica del sistema binario tales como "y", "o", etc. Gracias al rigor del sistema de Boole es posible mecanizar la lógica que opera sobre dos estados 0 y 1 , los cuales se nombraron anteriormente).

Desde comienzos de la segunda guerra mundial, se le confiỏ al húngaro Alan Turing, que se encontraba en Inglaterra desde 1936, la forma de descodificar los mensajes alemanes, Turing había precisado la noción de calculabilidad y adoptado la noción de algoritmo al cálculo de ciertas fun- ciones. También antes de la guerra, había definido la máquina de Turing, teóricamente capaz de calcular toda función calculable.

A mediados de los años cuarenta, John von Neuman, destacado matemático y miembro del Instituto de Escudios Avanzados de Princeton, New Jersey, en colaboración con H. H. Goldstine y A.W. Bruks, escribió una ponencia en la que sugirió que: 1) Los sistemas de numeración binaria fueran usados en la construcción de computadores, y 2) que las instrucciones de computadores, así como los datos procesados, fueran almacenados en una máquina. Estas ideas llegaron a ser parte básica del principio funcional de los computadores. Como el sistema binario de dos dígitos y los componentes electrónicos esta en una de dos condiciones (encendido o apagado).

A comienzos de los años cincuenta, dos importantes descubrimientos en ingeniería cambiaron la imagen de los consputadores electrónicos; de una máquina rápida con un hardware poco confiable se pasó a un computador de relativamente alta confiabilidad y aún mayor capacidad. Esos descubrimientos fueron la memoria de núcleo magnético y los circuitos de transistores.

\section{INTELIGENCIA ARTIFICIAL}

En 1964 lizo su aparición la inteligencia artificial, disciplina que consiste en construir programas de computador que tienden a imitar la inteligencia humana. Mientras la iuformática manipula mecánicamente la información, la inteligencia artificial formaliza el conocimiento y razonamiento hunanos. Según esta definición, se podría remontar el comienzo de la inteligencia artificial al año 450 a.C., época ел la que los grandes filósofos gijegos, como Sócrates, vislumbraban la idea de reducir codo razo. namiento a un cálculo, a semejanza de la geometría.

Con el desarrollo de la inteligencia artificial se abre el espacio para la producción de software especializado que "aprende de sí mismo" y este 
tipo de programas forma parte de la cotidianidad de universidades desarrolladas.

\section{SISTEMAS EXPERTOS}

La inteligencia artificial dio origen a los sistemas expertos, en la década del setenta. Un sistema experto es un programa que se caracteriza por su capacidad de razonamiento por inferencia lógica a partir de un problema plinteido por el usuario. El sistema se sirve de una base de conocinientos y de un conjunro de reglas, denominadas de producción, constituidas por un experto hunano.

\section{MICROCOMPUTADORES}

\section{COMPUTADORES PERSONALES}

En los años ochenta, las técnicas de integración a muy gran escala, permitieron colocar cientos de miles de transistores en un chip simple, continuando la tendencia a reducir los tamaños; esto llevó a la introducción de los microcomputadores o computadores personales, máquinas programables y de costo accesible para un usuario conuún y corriente.

Todo el campo de los computadores continúa siendo una grande y trenenda experiencia de adelanto y desarrollo; como la tecnología computacional y la de las comunicaciones continúan integrándose las redes de computadores, el correo electrónico y las publicaciones electrónicas sólo son el abrebocas de posteriores aplicaciones En nuestro país la presencia de los computadores se ha dado a través de las facultades de Ingeniería, lo cual ha craído como consecuencia que se les oriente hacia la urilización práctica de los programas existentes.

\section{LA BANDA ANCHA}

En los últimos 50 años se han hecho valiosos aportes para el desarrollo de la ingeniería química, la biología, las comunicaciones, la cibernética, la realidad virtual, etc. Con las comunicaciones se maneja mucha información, datos estadísticos y a rravés de la Internet y los teléfonos celulares podemos comunicación al instante, incluso con redes de información mundial.

Los servicios de comunicación tienden a ser desarroliados día a día con una mayor eficacia, por to que una formación sólida en las ciencias que se involucran en la ingeniería dan una mejor ayuda en los diferentes desarrollos tecnológicos del mundo, en un informe especial sobre nuevas directrices para el desarrollo de conexiones a alta velocidad en las empresas. La banda ancha hace su aparición: "En Colombia, la compañía Cilat ofrece servicios de conexión inalánbrica vía satélite a través de VSAT (Very Small Aperture Terminals) una solución inalámbrica, pero no móvil, ... Rolando Fernández, gerente general de Gilat, manifiesta que: 'con la llegada de la banda ancha, las empresas y corporaciones podrán materializar inmediatamente aplicaciones como videoconferencia entre cualquier par de oficinas, comunicaciones corporativas de telefonía solsre IP y LAN de alta velocidad. Las universidades pueden lograr sedes virtuales a distancia, con comunicación directa entre profesor- alumno'. Precisanrente, tocando la parte de educación. Gilat ha insplementado para el proyecto Conpartel, del ministerio de Comunicaciones en Colombia, 670 puntos de Internet con alta capacidad que benefician a las comunidades rurales con la comunicación y el acceso a la información obtenidos a través de Internet mediance el producto Skystar Advanrage".

\section{SITUACIÓN ACTUAL}

La díferencia, que en la actualidad se puede encontrar entre un técnico y el ingeniero, se tiene principalmente en la formación básica que reciben. En los dos primeros años de su carrera como ingeniero debería recibir formación en matemáticas, fisica. biología y química, para adquirir cualidades que le permitan interpretar y analizar datos que más adelante pueda conseguir y de esta manera se pueda enfrentar con éxito al desarrollo profesional, 
adquiriendo una actitud investigadora que le pernita hacer propuestas claras de mcjormmiento de proceso a todo nivel, reforzando con esto la formación clara en las ciencias básicils, mientras que al técnico no le interesa la parte formatival porque se dirige más a lo práctico. que es la directriz de las escuelas tecnológicas.

Las asignacuras que componen la formación del Ingeniero, cubren temas fundamentales que son de propósito general, independiencemente del objeco de estudio de cada carrera. Incluso los nombres coinciden en muchos casos y los remas cubicitos son similares. Se puede decir a grandes rasgos cuue esta formación incluye el estudio de las funciones de valor real en una y varias variables. el estudio de las estructuras de espacio vectorial, la introducción a la modelización con las ecuaciones diferenciales, así como tanmbién la progranzación lineal en la toma de decisiones, si es que se desea optinizar recursos y procesos, y no es "a ojo", como se realizan los pronósticos a largo plazo, sino con la técnica llamada re-gresión o ajuste de puntos en una serie de tiempo, que se puede estudiar en la asignatura de Escadística. En el caso de la Ingeniería de Sistenas también se deben incluir espacios de fornación en lógica matemácica que es la base de la programación.

\section{CONCLUSIONES}

A través de la historia la matemática, ha jugado un papel importante en rodos los descubrimientos hechos por el hombre y en la úlcima tecnología está a través de los ingenieros, cuyas bases matemáticas han sido sólidas y se han sabido utilizar en ella. De ésta forma la interdisciplinariedad nos dará nuchos y grandes avances.

Nos hemos venido navegando a través del tiempo desde la prehistoria; ha habido creaciones, inventos lasta la última tecnología hecha por el hombre. donde la matemática sienupre se ha hecho presente y seguirá estando aluí, en todo lo que el hombre se imagine construir o hacer para su beneficio.

\section{BIBLIOGRAFÍA}

alvarajo gamboa, Martha. Hitos del desarrollo de la matemática y la informática en Colombia y en el mundo. Universidad Javeriana,1999.

Baca Urbina. Gabriel. Introducción a la ingeniería, México, 1990.

Carrizosa, Valenzuela, Jullo. "La importancia de la matemática en la vida moderna", en Lecturas Matemáticas, discurso inaugural de la Sociedad Colombiana de Matenráticas (junio de 1956), Vol. 19., 1998, páginas 137-152.

Castro, Chadid Iván. El cálculo en Colombia. Presente pasado y futuro. Grupo Editorial Iberoamérica, 1997.

Consejo de Acreditación para la Ingeniería y la Tecnología de Estados Unidos de Norre América.

Dominguez Cajeli. Darío (et al) y Palacio Duque, Luz. Elena. Estimación de la dimensión fractal y su aplicación en fisica de suelos, 1999.

- Análisis fractal de la línea de base de macrófagos control y macrófagos infectodos con Leishmania Amozonensis. 2001.

Gonzílez G., Álvaro J. Prospectiva tecnológica de la ingenieria civil en Colombia. htcp:/www.icfes.gov.co revistas/ingeinve/n ${ }^{\circ} 37 /$ Art 2 .html

Ir Manager Banda Ancha, página 37, marzo 2002.

Vasco U., Carlos E. La informárica en los programas de matemáticas de la educación básica. Colciencias. 1988.

Velasco, Aurora. introducción a la ingeniería civil. Módulo para Educación a Distancia del programa de Ingeniería Civil, inédito, 2002. 